\title{
Primary Embryonal Lung Carcinoma and Testicular Seminoma in the Same Patient Ten Years Later: Case-Report and Literature Review"
}

\author{
Pasquale Rescigno, Giuseppe Di Lorenzo, Lucia Raimondo, Carmine D’Aniello, Elide Matano, \\ Vincenzo Damiano, Lucia Nappi, Livio Puglia, Antonella Petremolo, Piera Federico, \\ Giovannella Palmieri \\ Genitourinary Oncology and Rare Cancer Center, Federico II University, Naples, Italy \\ Email: giuseppedilorenzoncol@hotmail.com
}

Received April 1, 2012; revised April 9, 2012; accepted April 27, 2012

\begin{abstract}
Primary germ cell tumors of lung are extremely rare. The prognosis is usually poor, with various symptoms seriously affecting quality of life. In this paper we describe the unique case of a patient affected by an embryonal carcinoma of lung and a testicular seminoma after ten years. We also report literature about pulmonary extragonadal germ cell tumors.
\end{abstract}

Keywords: Extragonadal Germ Cell Tumors; Primary Pulmonary Germ Cell Tumor; Embryonal Carcinoma; Testicular Seminoma

\section{Introduction}

Primary germ cell tumors originating in the lung are extremely rare. In adults, the most common sites of extragonadal germ cell tumors are the mediastinum, retroperitoneum, and cranium; they histologically comprise seminomas $(30 \%-40 \%)$ and nonseminomatous tumors $(60 \%$ - 70\%) NSGCTs (nonseminomatous germ cell tumors) [1]. The prognosis is usually poor, with various symptoms seriously affecting quality of life [1].

In this paper we describe the case of a patient affected by an embryonal carcinoma of lung and a testicular seminoma after ten years and its treatment. We also report literature about pulmonary extragonadal germ cell tumors to elucidate its features, survival outcome and best management.

\section{Case Report}

In august 2002 a man 42 years old arrived at our institution with following symptoms: chest pain, dyspnea, as thenia and weight loss. He didn't refer to serious illnesses at history. So we decided to practice blood count, biochemical exams and chest Radiography.

Blood count and biochemical exams were normal, except for LDH (1315 UI/L). Chest radiography showed a large mass in left middle-lower lobe (Figure 1). To invest-

${ }^{*}$ Conflict of interest: none to declare. tigate further patient performed computed tomography (CT) scan whole-body (w.b.), which revealed large (10.0 $\times 7.0 \mathrm{~cm}$ ) heterogeneous solid mass in the left mid-lung posteriorly, involving the lower lobe (Figure 2), no lymph nodes seemed to be involved.

On the basis of this radiological a FNAC (fine needle aspiration cytology) was carried out on the left lung mass. It showed clusters of big epithelial-like cells in a background of extensive necrosis; so it was interpreted as adenocarcinoma with necrosis (Figure 3). In September 2002 the patient underwent left pneumectomy with lymphadenectomy. Histological examination of lung mass presented

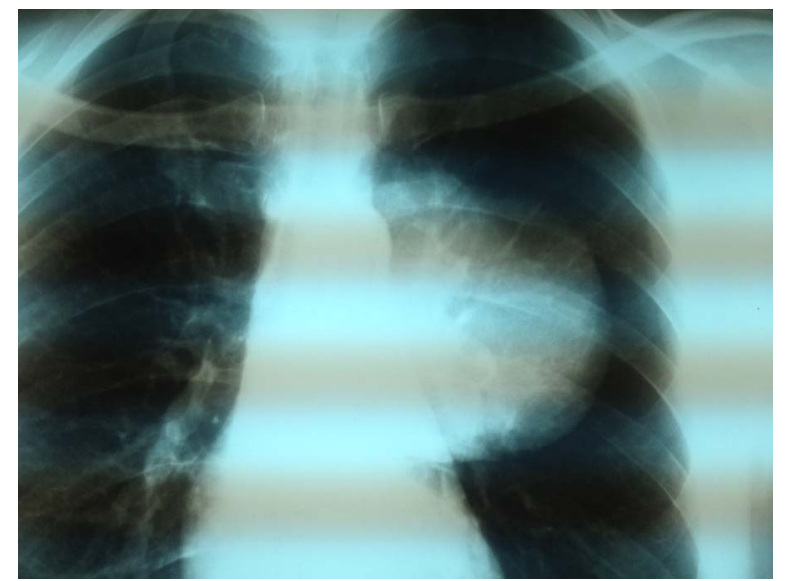

Figure 1. Chest X-ray detecting lung mass. 


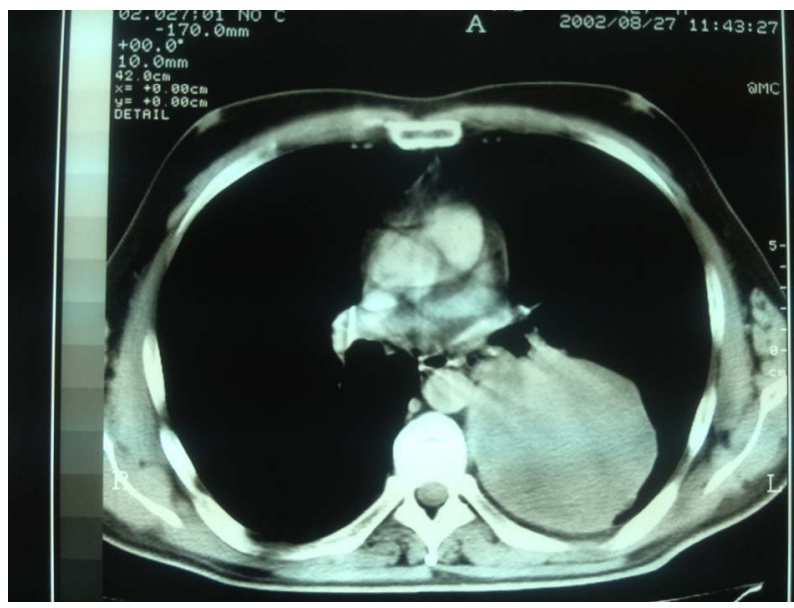

Figure 2. Chest CT.

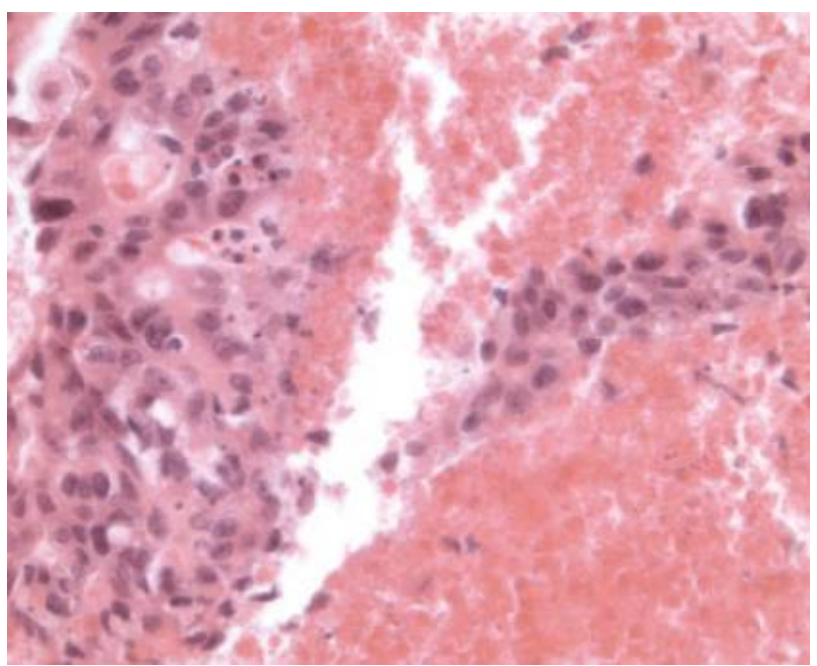

Figure 3. FNA of the lung mass showing groups of malignnant cells associated with necrosis (Hematoxylin and Eosin, $\times 400)$.

poorly differentiated epithelial neoplasm, with focal glandular aspects.

Immunohistochemistry stains on the cell block were positive for cytokeratin (CK), Placental Alk. Phosphatase (PLAP), CD30 and negative for alpha fetoprotein (AFP), Thyroid Transcription Factor-1 (TTF-1), beta human chorionic gonadotropin (BhCG), c-Kit. At immunochemistry PLAP and CD30 are commonly positive in germ cell cancer, negativity to TTF-1 excludes the possibility of a metastatic lesion and directs to a primary lesion of lung.

So these elements were compatible with no-small lung cancer or germ cell tumor. Review of slides confirmed presence of big cells neoplasm with a solid pattern, focal glandular aspects and large areas of necrosis; these cells had ample cytoplasm, large nuclei with multiple macronucleoli. Immunohistochemical analysis validated the diagnosis of embryonal carcinoma. So patient performed testicular echography and an evaluation of tumor markers, both of them resulted negative. Therefore four courses of BEP (bleomycin $30 \mathrm{mg} \mathrm{2,} \mathrm{9,} \mathrm{16;} \mathrm{etoposide} 100 \mathrm{mg} / \mathrm{mq} \mathrm{1}$; cisplatin $20 \mathrm{mg} / \mathrm{mq} 1 \mathrm{q} 21$ ) were applied as adjuvant treatment. The patient was then followed up for eight years with no evidence of disease at instrumental and biochemical controls.

However in april 2011 level of bHCG started increasing so a new ultrasound of testis was performed and a right testicular mass was detected (Figure 4). A CT scan w.b. was carried out, distant metastases were not found. Patient underwent orchiectomy. Histological examination confirmed the presence of classical seminoma. It was large $2 \mathrm{~cm}$. Tunica albuginea, vaginalis and cord were free from invasion and there weren't neoplastic emboli.

Immunohistochemistry was positive for PLAP and negative for pan-CK, $\alpha \mathrm{FP}, \beta \mathrm{HCG}$.

After surgery CT scan was negative and tumor markers were normal so we decided with patient and psychologist to start surveillance.

\section{Discussion}

Extagonadal germ cell tumors are rare, they account for $1 \%-5 \%$ of all germ cell tumors [2] but primary pulmonary location of germ cell tumor is extremely rare, few cases of yolk sac tumors [3-6] and about 40 cases of choriocarcinomas (PPC) have been reported in literature.

In 2004 Umemori and his colleagues, as first, reviewed 25 previously described cases of primary pulmonary choriocarcinoma (PPC) [7]. He observed that PCC was more common in women than in men, who were generally older. The median survival time was 5.0 months and 1-, 2- and 5-year survival rates of $41 \%, 34 \%$ and $34 \%$, respectively. Survival for patients without metastases at presentation was longer than for patients with metastases $(p=0.019)$. Patients treated with both surgery and chemotherapy survived longer than those treated with either

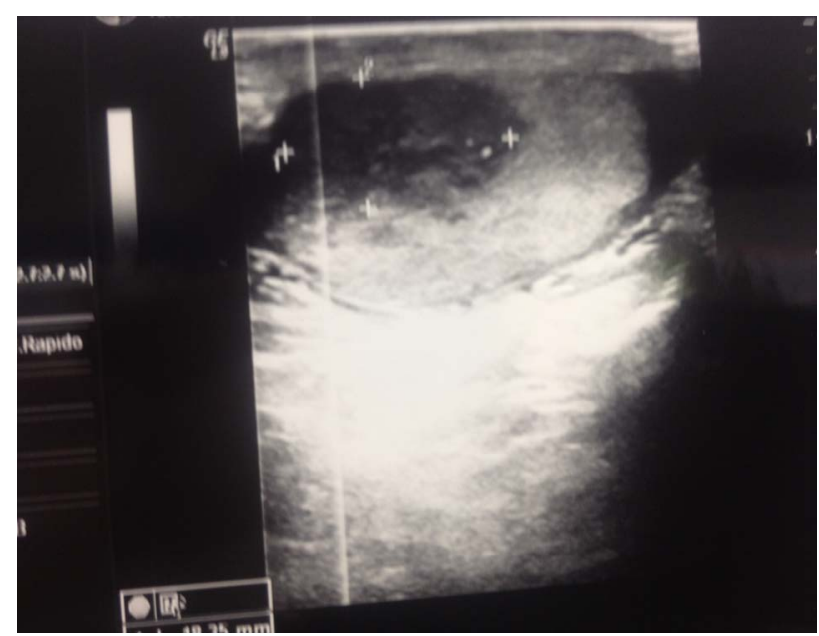

Figure 4. Testicular ultrasound detecting right mass. 
alone, or with optimal supportive care $(\mathrm{p}=0.038)$. Patients with smaller tumors $(<5 \mathrm{~cm})$ tended to survive longer than patients with larger tumors, but with short statistical significance $(\mathrm{p}=0.052)$ [7].

In the same year, Vaideeswar et al. reported seven cases of PPC seen in autopsy and surgical materials [8]. Four additional cases were found in a review by Arabi and collegues [9]. In our review of the literature to date, we found four additional cases of PPC [10]. All these reviews confirm data reported by Unemori.

Smaller number of cases has been reported as mixture of choriocarcinoma with adenocarcinoma elements [1113]. These cases are mostly revisions of autopsy materials. They are often confused with lung adenocarcinomas on FNA. Both may have similar cellular arrangements and similar cytomorphology. Both can show a background of tumor necrosis, as in our case [9].

From the above it's clear that is difficult to make diagnosis of a primary germ cell tumor of lung and to differentiate it from an adenocarcinoma based on both frequency and histological exam. However other elements can be helpful as in our case.

First of all when a diagnosis of an extragonadal germ cell tumor is supposed, it must be considered metastasis from occult or "burned out" gonadal cancer (a regressedtumor) until proved otherwise [14]. So a testicular ultrasound is recommended because a burned out gonadal primary tumor that presents with a metastasis is seen as an echogenic scar or a hypoechoic tissue on ultrasound [15]. In our case testicular ultrasound didn't show these features, so lung mass cannot be considered as a metastasis of a burned out gonadal cancer. Tumor markers are helpful in the diagnosis and follow-up of extagonadal germ cell tumors. Levels of $\beta$-HCG can be elevated in choriocarcinoma, embryonal carcinoma, and in about $10 \%$ of cases of seminomas, while levels of $\alpha$-fetoprotein can be elevated in endodermal sinus tumors and embryonal carcinoma [16]. Moreover nonseminomatous tumor diagnosis is possible without histopathological confirmation when the AFP level is over $500 \mathrm{ng} \cdot \mathrm{mL}^{-1}$. However, primary lung cancers that produce AFP have been reported [3].

As regard imaging features on $\mathrm{CT}$ scan, seminomas are usually bulky lobulated homogeneous mildly enhancing masses on CT. Cysts, hemorrhage and calcification are rarely seen. Instead NSGCTs (non seminomatous germ cell tumors) are usually large, lobulated, and heterogeneous, with calcification, hemorrhage, and necrosis [17]. So it's clear that CT features of mass we detected corresponded to NSGCT characteristics (Figure 2).

Also immunochemistry can help to make diagnosis of extragonadal cancer cell tumor. Usually germ cell tumors (both seminoma and NSGCTs) highly express monoclonal PLAP. However while embryonal carcinoma more often express cytokeratins (especially CD30), seminoma stained positively in a minority of cases [18]. These characteristics led us to diagnosis of embryonal cancer in 2002 and seminoma in 2011.

Here we have showed all elements that led us to this difficult diagnosis, but decision about treatment hasn't been easier. Because of the extreme rarity of this malignnancy, there are no standardized therapeutic guidelines. In all cases we reported here resection followed by adjuvant chemotherapy is represented as the most effective treatment and these were the recommendations we followed.

\section{Conclusions}

The case that we reported is the first in literature of lung embryonal carcinoma and a rare case of extragonadal germ cell tumor which has developed a testicular seminoma after ten years.

Moreover unlike other types of NSGCTs embryonal carcinoma seems to be less aggressive with a long life expectancy after an adjuvant treatment.

Unfortunately few data we have doesn't allow us to draw definitive evidence. We hope international cooperation can make available more data about extragonadal germ cell tumors.

\section{REFERENCES}

[1] C. Bokemeyer and C. R. Nichols, "Extragonadal Germ Cell Tumors of the Mediastinum and Retroperitoneum: Results from an International Analysis," Journal of Clinical Oncology, Vol. 20, No. 7, 2002, pp. 1864-1873. doi:10.1200/JCO.2002.07.062

[2] J. K. McKenney and A. Heerema-McKenney, "Extragonadal Germ Cell Tumors: A Review with Emphasis on Pathologic Features, Clinical Prognostic Variables, and Differential Diagnostic Considerations," Advances in Anatomic Pathology, Vol. 14, No. 2, 2007, pp. 69-92. doi:10.1097/PAP.0b013e31803240e6

[3] A. Basoglu and A. T. Sengul, "Pure Yolk-Sac Tumor of the Lung," Asian Cardiovascular and Thoracic Annals, Vol. 16, No. 5, 2008, pp. 410-411.

[4] H. Inoue and M. Iwasaki, "Pure Yolk-Sac Tumor of the Lung," Thoracic and Cardiovascular Surgery, Vol. 41, No. 4, 1993, pp. 249-251. doi:10.1055/s-2007-1013864

[5] N. Kakkar and R. K. Vasishta, "Primary Pulmonary Malignant Teratoma with Yolk Sac Element Associated with Hematologic Neoplasia," Respiration, Vol. 63, No. 1, 1996, pp. 52-54. doi:10.1159/000196516

[6] G. Pelosi and F. Petrella, "A Primary Pure Yolk Sac Tumor of the Lung Exhibiting CDX-2 Immunoreactivity and increased Serum Levels of Alkaline Phosphatase Intestinal Isoenzyme," International Journal of Surgical Pathology, Vol. 14, No. 3, 2006, pp. 247-251. doi:10.1177/1066896906290657

[7] Y. Umemori and A. Hiraki, "Primary Choriocarcinoma of the Lung," Anticancer Research, Vol. 24, No. 3B, 2004, pp. 1905-1910. 
[8] P. Vaideeswar and J. Mehta, "Primary Pulmonary Choriocarcinom-A Series of 7 Cases," Indian Journal of Pathology and Microbiology, Vol. 47, No. 4, 2004, pp. 494496.

[9] H. Arabi and M. Shah, "Aspiration Biopsy Cytomorphology of Primary Pulmonary Germ Cell Tumor Metastatic to the Brain," Diagnostic Cytopathology, Vol. 37, No. 10, 2009, pp. 715-719. doi:10.1002/dc.21089

[10] I. Maestá and F. V. Leite, "Primary Pulmonary Choriocarcinoma after Human Chorionic Gonadotropin Normalization Following Hydatidiform Mole: A Report of Two Cases," Journal of Reproductive Medicine, Vol. 55, No. 7-8, 2010, pp. 311-316.

[11] H. Adachi and T. Aki, "Combined Choriocarcinoma and Adenocarcinoma of the Lung," Acta Pathologica Japonica, Vol. 39, No. 2, 1989, pp. 147-152.

[12] S. Yamamoto and H. Tanaka, "Primary Pulmonary Choriocarcinoma Combined with Adenocarcinoma," Pathology International, Vol. 56, No. 7, 2006, pp. 402-407. doi:10.1111/j.1440-1827.2006.01977.x

[13] F. Chen and A. Tatsumi, "Combined Choriocarcinoma and Adenocarcinoma of the Lung Occurring in a Man:
Case Report and Review of the Literature," Cancer, Vol. 91, No. 1, 2001, pp. 123-129. doi:10.1002/1097-0142(20010101)91:1<123::AID-CNCR 16>3.0.CO;2-3

[14] B. Coulier and Y. Lefebvre, "Metastases of Clinically Occult Testicular Seminoma Mimicking Primary Extragonadal Retroperitoneal Germ Cell Tumors," Journal Belge de Radiologie, Vol. 91, No. 4, 2008, pp. 139-144.

[15] J. C. Angulo and J. González, "Clinicopathological Study of Regressed Testicular Tumors (Apparent Extragonadal Germ Cell Neoplasms)," Journal of Urology, Vol. 182, No. 5, 2009, pp. 2303-2310. doi:10.1016/j.juro.2009.07.045

[16] A. B. Shinagare and J. P. Jagannathan, "Adult Extragonadal Germ Cell Tumors," American Journal of Roentgenology, Vol. 195, No. 4, 2010, pp. 274-280.

[17] R. G. Levitt and J. E. Husband, "CT of Primary GermCell Tumors of the Mediastinum," American Journal of Roentgenology, Vol. 142, No. 1, 1984, pp. 73-78.

[18] J. C. Cheville and S. Rao, "Cytokeratin Expression in Seminoma of the Human Testis," American Journal of Clinical Pathology, Vol. 113, No. 4, 2000, pp. 583-588. 